\title{
Universiteit
}

Leiden

The Netherlands

\section{Predation risk constrains herbivores' adaptive capacity to warming}

Veldhuis, M.P.; Hofmeester, T.R.; Balme, G.; Druce, D.J.; Pitman, R.T.; Cromsigt, J.P.G.M.

\section{Citation}

Veldhuis, M. P., Hofmeester, T. R., Balme, G., Druce, D. J., Pitman, R. T., \& Cromsigt, J. P. G. M. (2020). Predation risk constrains herbivores' adaptive capacity to warming. Nature Ecology And Evolution, 4, 1069-1074. doi:10.1038/s41559-020-1218-2

Version:

Publisher's Version

License:

Licensed under Article 25fa Copyright Act/Law (Amendment Taverne)

Downloaded from: https://hdl.handle.net/1887/3200771

Note: To cite this publication please use the final published version (if applicable). 


\title{
Predation risk constrains herbivores' adaptive capacity to warming
}

\author{
Michiel P. Veldhuis ${ }^{1,2,3 凶}{ }^{\infty}$, Tim R. Hofmeester ${ }^{4}{ }^{4}$, Guy Balme ${ }^{5,6}$, Dave J. Druce ${ }^{7,8}$, Ross T. Pitman ${ }^{5,6}$ \\ and Joris P.G.M. Cromsigt (i) $4,9,10$
}

\begin{abstract}
Global warming compels larger endothermic animals to adapt either physiologically or behaviourally to avoid thermal stress, especially in tropical ecosystems. Their adaptive responses may however be compromised by other constraints, such as predation risk or starvation. Using an exceptional camera-trap dataset spanning 32 protected areas across southern Africa, we find that intermediate-sized herbivores $(100-550 \mathrm{~kg})$ switch activity to hotter times of the day when exposed to predation by lions. These herbivores face a tight window for foraging activity being exposed to nocturnal predation and to heat during the day, suggesting a trade-off between predation risk and thermoregulation mediated by body size. These findings stress the importance of incorporating trophic interactions into climate change predictions.
\end{abstract}

T he earth is experiencing a major defaunation crisis and is rapidly losing its remaining larger mammalian herbivores and carnivores $^{1}$. Habitat loss, hunting and conflict are ongoing major threats to the world's last truly diverse and abundant megafaunal (animals $>40 \mathrm{~kg}$ ) strongholds in Africa ${ }^{2,3}$. The remaining assemblages now increasingly face threats from climate change $e^{4}$, which is predicted to result in a high turnover in species composition across African systems due to species range shifts ${ }^{5}$. Although historically some species survived climate change by continent-scale tracking of suitable climates ${ }^{6}$, habitat degradation and human-made barriers increasingly prohibit range shifts by many larger mam$\mathrm{mals}^{7,8}$, increasing extinction risk ${ }^{9}$. We urgently need to understand how African megafauna may respond to global warming, given the restrictions in range shift options.

Maximum temperature across Africa is expected to rise faster than the global average ${ }^{10}$ and is an important direct thermal stressor in African savannas ${ }^{11,12}$. Mammals can reduce the effects of thermal stress through behavioural thermoregulation, such as moving to thermal refugia ${ }^{13}$ and/or changing activity to cooler times of day $^{14}$. Such behavioural adaptations are less costly than physiological adjustments ${ }^{15}$, and they are likely to be the primary response to changing climates because rapid adaptation through genetic change is improbable given the relatively long generation time of large mammals ${ }^{11}$. Despite this, temporal shifts in activity times as a response to climate change have been poorly studied (but see refs. $\left.{ }^{16-18}\right)$.

Temperature varies over the day, producing a 'timescape of heat' that provides options for animals to modify their behaviour temporally as a way to reduce heat stress. However, these options might be compromised by other constraints, specifically predation risk ${ }^{19}$ and food requirements ${ }^{12}$. Predator activity or hunting success may vary predictably on a daily basis, resulting in a concurrent 'timescape of risk, where species can adaptively respond to temporal patterns of predation risk ${ }^{17,20}$. In contrast, food availability for herbivores is relatively constant throughout the day, so that large herbivores can choose when to forage, trying to avoid the hot and risky moments of the day, while still meeting their food requirements. The responses of large mammals to the trade-offs between thermoregulation, predation risk and food requirements remain unexplored ${ }^{21}$.

Here, we provide empirical evidence that carnivores limit the capacity of their ungulate prey to adapt to warmer conditions by comparing herbivore diel activity patterns across sites with and without lions in South Africa. The country hosts many protected areas (PAs) that vary in climatic conditions and large carnivore presence due to a history of local megafauna extirpations and reintroductions. We used this unique opportunity, in combination with an unprecedented countrywide multi-annual camera-trapping dataset, to investigate activity patterns of savannah herbivores. Seventy-three surveys (average length $50 \mathrm{~d}$ ) were conducted across 32 PAs that spanned a strong temperature gradient (mean daily temperature ranged from 11.8 to $27^{\circ} \mathrm{C}$ between surveys). Lions (Panthera leo) were extirpated in all 32 PAs halfway through the twentieth century and later on reintroduced in half of them $(n=16 \mathrm{PA})$ yielding a 'natural experiment' with contrasting lion presence that is independent of environmental conditions (Extended Data Fig. 1 and Supplementary Table 1). Leopard (Panthera pardus) and spotted hyena (Crocuta crocuta) were present in most PAs (30 and 24 respectively) but were not reintroduced so that their distributions are not independent from environmental conditions. Cheetah (Acinonyx jubatus) occurred in 20 PAs and African wild dog (Lycaon pictus) in 17 but at much lower densities (Extended Data Fig. 3). The low sample sizes and confounding factors prevented us from experimentally testing for effects of these smaller carnivores. We first used data for all carnivores together to investigate the relationships between activity, general predation risk and temperature. Subsequently, we used the 'natural experiment' to further explore the risk-temperature trade-off by testing how lion

\footnotetext{
Institute of Environmental Sciences, Leiden University, Leiden, the Netherlands. ${ }^{2}$ Department of Ecology and Evolutionary Biology, Princeton University, Princeton, NJ, USA. ${ }^{3}$ Groningen Institute for Evolutionary Life Sciences, University of Groningen, Groningen, the Netherlands. ${ }^{4}$ Department of Wildlife, Fish, and Environmental Studies, Swedish University of Agricultural Sciences, Umeå, Sweden. ${ }^{5}$ Panthera, New York, NY, USA. ${ }^{6}$ Institute for Communities and Wildlife in Africa, Department of Biological Sciences, University of Cape Town, Rondebosch, South Africa. ${ }^{7}$ Ezemvelo KZN Wildlife, Cascades, South Africa. ${ }^{8}$ School of Life Sciences, University of KwaZulu-Natal, Scottsville, South Africa. ${ }^{9}$ Department of Zoology, Nelson Mandela Metropolitan University, Port Elizabeth, South Africa. ${ }^{10}$ Environmental Sciences Group, Copernicus Institute of Sustainable Development, Utrecht University, Utrecht, the Netherlands. 凶e-mail: m.p.veldhuis@cml.leidenuniv.nl
} 
$\mathbf{a}$
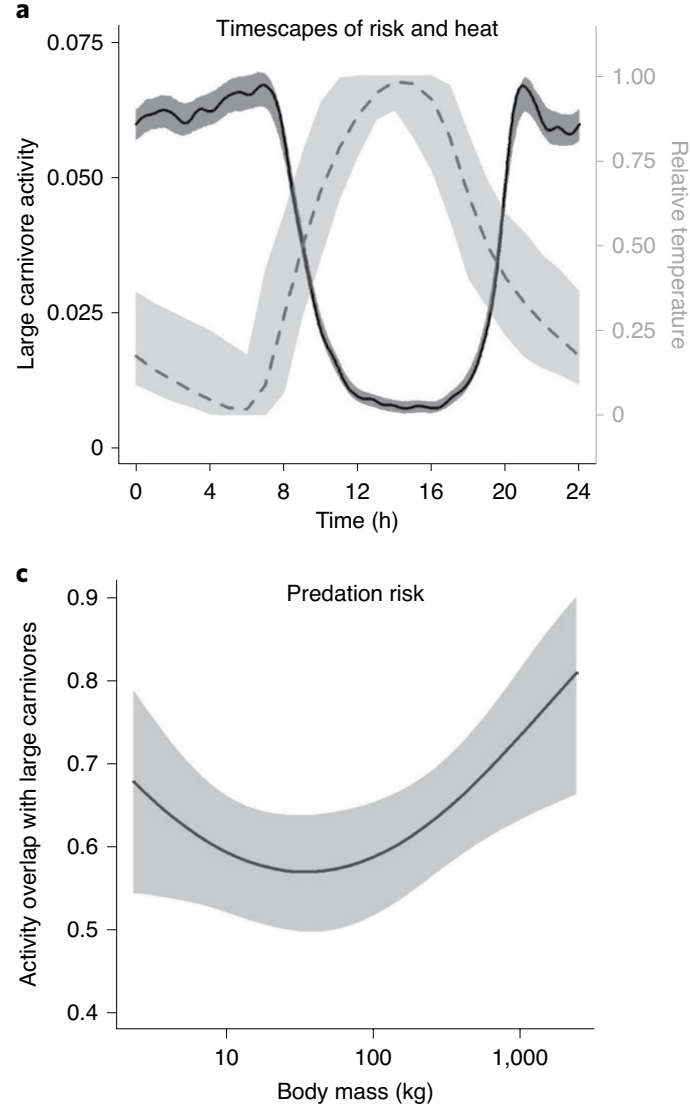

b
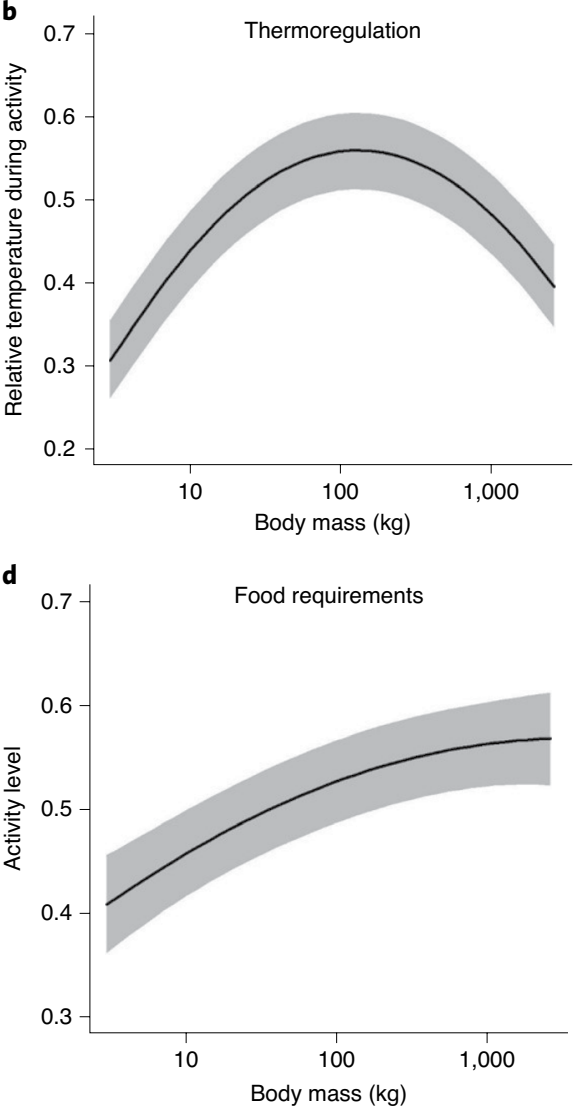

Fig. 1 | Herbivore constraints in African savannas. a-d, Timescapes of heat ( $n=19,200$ temperature measurements across 73 surveys) and risk ( $n=16,059$ detections for five large carnivores) (a), and the general relationships between herbivore body mass and thermoregulation (b; $n=141,883$ detections for 29 herbivores), predation risk (c; $n=141,883$ detections for 29 herbivores) and food requirements ( $\mathbf{d} ; n=141,883$ detections for 29 herbivores). Activity patterns (timing, overlap and level) were quantified using camera-trap detections. The dotted grey line in a represents average relative temperatures between sites and their minimum and maximum (light grey area). The solid black line in a represents the activity kernel with its $95 \%$ confidence interval (dark grey area). The lines in $\mathbf{b}, \mathbf{c}$ and $\mathbf{d}$ represent GLMM predictions with their $95 \%$ confidence interval (grey area).

presence affected the diel activity patterns of their prey, and more specifically, the average temperature of the time at which prey are active. A total of 141,883 independent detections yielded sufficient data ( $>100$ detections per survey) for 29 herbivores spanning more than three orders of magnitude in body mass, from $3-\mathrm{kg}$ scrub hare (Lepus saxatilis) to 4,000-kg elephant (Loxodonta Africana) (Supplementary Table 2).

Body size is a key trait that influences thermoregulation ${ }^{22}$, predation risk ${ }^{23}$ and food requirements ${ }^{24}$. Body size is thus expected to act as the unifying currency to predict responses of mammalian herbivores to warming while avoiding predation and starvation. Animals of larger body size have a smaller surface to volume ratio, resulting in decreased capacity to dissipate excess heat through the skin or extremities ${ }^{25}$. Similarly, nutritional requirements increase with body size ${ }^{24}$, so that larger animals must compensate by increasing their food intake to meet their increased nutritional needs ${ }^{26}$. This requires increased foraging time and therefore prolonged periods of activity ${ }^{24}$. In contrast, predation risk decreases with large body $\operatorname{size}^{23}$. We expected that smaller species should be more responsive to predation risk, whereas larger species should be more concerned about finding sufficient food while avoiding activity at times of the day when dissipating metabolic heat is challenging.

To evaluate our general predictions regarding herbivore body size, we initially quantified differences in diel activity patterns relative to temperature, predation risk and food requirement using the entire dataset for all 29 species. To investigate how body size affects selection for activity during cool periods (thermoregulation), we quantified the daily timescape of heat (Fig. 1a and Extended Data Fig. 2) using hourly temperature data from 17 weather stations located in near the protected areas (Extended Data Fig. 1). To allow comparisons between surveys we rescaled temperatures so that 0 and 1 would indicate the time of lowest and highest temperature in each site respectively, and determined the relative temperature for each camera-trap detection, representing the thermal selection timescape for herbivores. To test for a relationship between body size and response to predation risk, we quantified a timescape of risk (Fig. 1a and Extended Data Fig. 3) using combined diel activity distribution of the larger carnivores and determined overlap in activity with each herbivore species. The temporal overlap between predators and their prey directly determines the strength of their interspecific interaction ${ }^{27}$. To investigate how food requirements depend on body size, we calculated animal activity levels on the basis of the evenness of the distribution of animal detections across the day ${ }^{28}$, assuming most of the activity is taken up by foraging or moving between foraging patches.

\section{Results}

Large carnivores were most active during the coolest hours of the day resulting in reversed timescapes of risk and heat (Fig. 1a and Extended Data Figs. 2 and 3). Small herbivores $(<40 \mathrm{~kg})$ and megaherbivores $(>700 \mathrm{~kg})$ were mostly active during times with relatively low temperatures (generalized linear mixed model, 


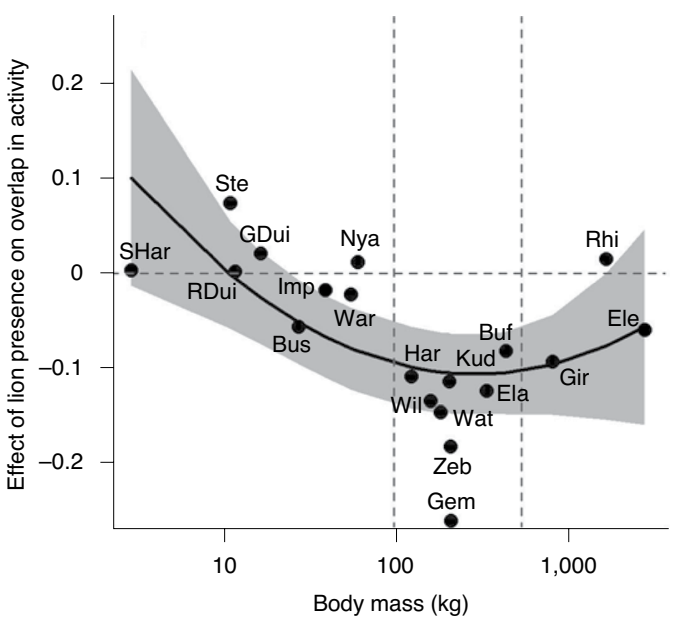

b

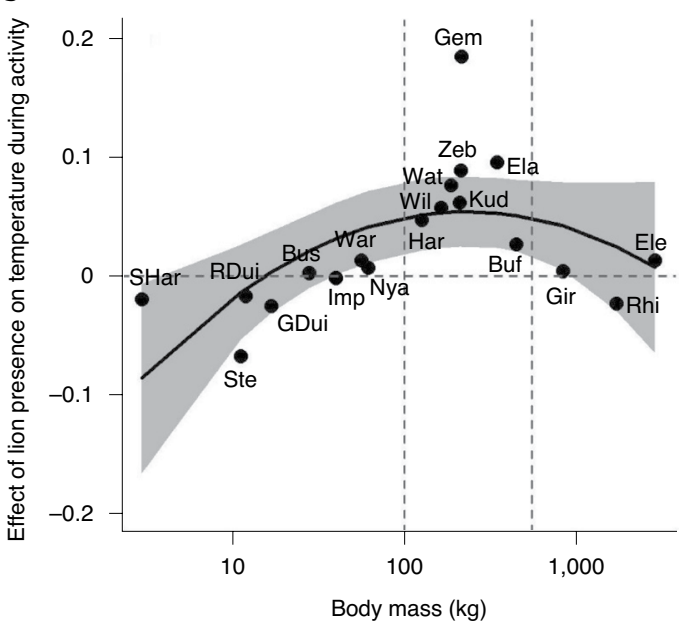

Fig. 2 | Lion presence changes herbivore activity patterns. a,b, Lion presence shifts diel activity patterns of intermediate-size herbivores (100-550kg) to reduce the predicted overlap with lion activity (a) and increase in relative temperature during herbivore activity (b) relative to areas where lions are absent. Horizontal dotted line at 0 indicates no difference in diel activity (a) and temperature (b) between areas with and without lions present. SHar, scrub hare (Lepus saxatilis); Ste, steenbok (Raphicerus campestris); RDui, red duiker (Cephalophus natalensis); GDui, grey duiker (Sylvicapra grimmia); Bus, bushbuck (Tragelaphus scriptus); Imp, impala (Aepyceros melampus); War, common warthog (Phacochoerus africanus); Nya, nyala (Tragelaphus angasii); Har, hartebeest (Alcelaphus buselaphus); Wil, common wildebeest (Connochaetes taurinus); Wat, waterbuck (Kobus ellipsiprymnus); Kud, greater kudu (Tragelaphus strepsiceros); Zeb, plains zebra (Equus quagga); Gem, gemsbok (Oryx gazella); Ela, common eland (Tragelaphus oryx); Buf, buffalo (Syncerus caffer); Gir, giraffe (Giraffa camelopardalis); Rhi, white rhino (Ceratotherium simum); Ele, elephant (Loxodonta africana). Vertical lines delineate preferred prey range of lions (100-550kg). Black lines in $\mathbf{a}$ and $\mathbf{b}$ represent predictions from linear regression models and their $95 \%$ confidence interval (grey area) including a quadratic terms for body mass.

GLMM: $z=-15.4, P<0.001$ ) (Fig. 1b, Extended Data Fig. 4 and Supplementary Table 3), while temporal avoidance of large carnivores was greatest among herbivores of intermediate size (40$700 \mathrm{~kg}$ ) (GLMM: $z=-2.2, P=0.02$ ) (Fig. 1c and Supplementary Table 4). Activity levels increased with body mass (GLMM: $z=8.63$, $P<0.010)$ but levelled off with higher body mass $(z=-2.75$, $P<0.01$ ) (Fig. 1d and Supplementary Table 3). These results mostly followed our expectations, except for the smallest herbivores that were also mainly nocturnal (especially scrub hare and springhare, Pedetes capensis) or crepuscular (in particular red and grey duiker, Cephalophus natalensis and Sylvicapra grimmia) (Extended Data Fig. 4), probably to prevent dehydration ${ }^{18,29}$ and/or predation by diurnal predators, such as birds of prey.

To differentiate between the effects of temperature and predation, we compared diel activity patterns at sites with and without lions. Herbivore species that fall within the preferred prey range of lions (100-550 kg; Extended Data Fig. 5) strongly reduced their overlap with the times lions are most active, especially when lions were present (GLMM: $z=-4.5, P<0.001$ ) (Supplementary Table 4). At sites without lions, these herbivores became more active at times that lions would have been active; that is, they increased temporal overlap with lion activity projected from sites with lions at days with corresponding temperatures (linear model: $t=6.9, P<0.001$ ) (Supplementary Table 5 and Fig. 2a). Consequently, the relative temperature during activity for intermediate-sized herbivores was higher when lions were present (linear model: $t=-2.5, P=0.02$ ) (Supplementary Table 5 and Fig. 2b). Together, these results suggest that high ambient temperatures are a serious constraint for herbivores, particularly intermediate-sized herbivores $(100-550 \mathrm{~kg})$, and thus predation risk limits the behavioural capacity of herbivores to deal with high temperatures.

To investigate which species were most constrained by lion presence we calculated the change in overlap and relative temperature of activity as a result of lion presence (Fig. 2) and visualized the effects of lion presence on diel activity patterns for their preferred prey species (Fig. 3). Gemsbok (Oryx gazella) and plains zebra (Equus quagga) responded most strongly to lion presence (Fig. 2). Both species switched from diurnal activity with lions present to a nocturnal pattern with lions absent (Fig. 3e,f), reducing temporal overlap with lions when present by about $30 \%$ (from 0.6 to 0.4 ), at the cost during their activity of increased average temperature by more than $1{ }^{\circ} \mathrm{C}$. Two larger species, eland (Tragelaphus oryx) and buffalo (Syncerus caffer), were also mostly nocturnal when lions were absent (Fig. 3g,h). Eland increased diurnal activity when lions were present but not as strongly as zebra and gemsbok. Buffalo did not make a clear switch to diurnal activity when lions were present. Large herd size allows buffalo to defend themselves against lions ${ }^{31}$, potentially facilitating activity during cooler hours despite increased overlap with lion activity. The four smaller prey species were mostly active during daytime at sites with lions present (Fig. 3a-d). They all increased nocturnal activity and decreased activity during the hot midday when lions were absent but none became nocturnal. This lack of a clear switch to nocturnal activity may relate to these prey species also being preyed upon by other large carnivores, such as spotted hyena $^{32}$.

\section{Discussion}

Our results show that African ungulates face reversed timescapes of risk and heat, where the coolest times of the day equal those of highest carnivore activity and vice versa. The ecological implications of this trade-off between risk of predation and heat stress are mediated by body size. Balancing these risks becomes increasingly problematic for larger ungulates because higher food requirements force them to spend more of the day foraging while they are physiologically less capable of dissipating heat. However, this pattern changes when herbivores become too large to be caught, severely reducing (or eliminating) the predation risk constraint. It has been suggested that elephant and white rhino would perform all their feeding during the cooler night times, if they were not constrained by food ingestion and digestion rates ${ }^{24}$. Moreover, megaherbivores 

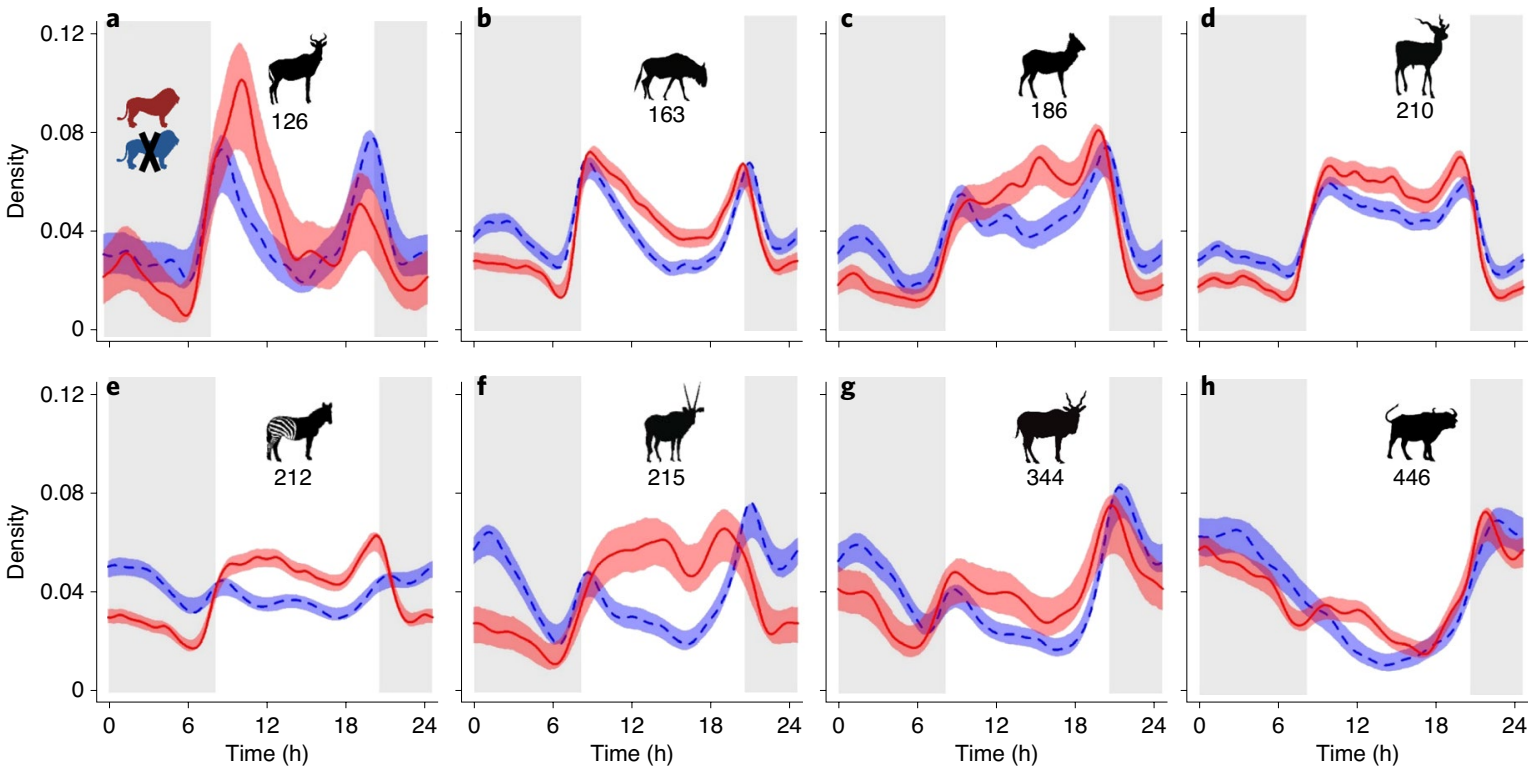

Fig. 3 Diel activity patterns in absence (blue) or presence (red) of lion (Panthera leo). a-h, Herbivores are ordered by average female body mass ${ }^{44}$ presented in kilograms below each silhouette: hartebeest (a; Alcelaphus buselaphus), common wildebeest (b; Connochaetes taurinus), waterbuck (c; Kobus ellipsiprymnus), greater kudu (d; Tragelaphus strespsiceros), plains zebra (e; Equus quagga), gemsbok (f; Oryx gazella), common eland (g; Tragelaphus oryx) and buffalo (h; Syncerus caffer). Lines represent activity kernels and their $95 \%$ confidence interval. Grey areas are times of high lion (and other carnivore) activity.

have been shown to prefer habitat with higher vegetation cover that can serve as thermal refugia but are perceived as risky and therefore avoided by smaller herbivores ${ }^{33,34}$ (but see ref. ${ }^{35}$ ).

Intermediate-sized herbivores face a tightening window for foraging activity being exposed to predation at night and to heat during the day. Increased nocturnal activity of intermediate-sized herbivores $(100-550 \mathrm{~kg})$ in areas without lions suggests substantial costs of this tightening window. On average, lions pushed intermediate-sized herbivores to be active at $~ 5 \%$ warmer times (Fig. 2b), which is on average about $0.75^{\circ} \mathrm{C}$ given the daily temperature variation (Extended Data Fig. 2a). Importantly, we found strong interspecific differences, where species such as gemsbok and zebra reduced their overlap with lion activity to a much larger extent, resulting in a much stronger increase in average temperature during activity, than for species such as buffalo and hartebeest. To be able to interpret the functional consequences of these differences, we need a much stronger quantitative understanding that integrates the effects of predation and thermoregulatory constraints to fitness parameters of these species. Our results suggest that such work is urgently needed as a foundation for accurate predictions of Africa's large herbivore populations under a future climate.

Food requirements, temperature and predation risk are general constraints in many ecosystems, although their ecological consequences may be different. The lower ambient temperatures in high-latitude ecosystems are expected to affect large herbivore behavioural adjustments (e.g. timing of activity) but the thermal constraints change from risk of increased heat stress to reduced cold stress compared to tropical systems ${ }^{22}$. Black-tailed deer (Odocoileus hemionus) increased diurnal activity in the absence of predators ${ }^{36}$ so that the top-down effects on ungulates in response to global warming could have radically different effects in ecosystems at higher latitude ${ }^{17}$. We suggest that predation risk can severely impact behavioural adaptive capacity, stressing the need to include trophic interactions in climate change predictions ${ }^{12}$, especially for endangered megafauna whose ranges are increasingly restricted by habitat fragmentation.

\section{Methods}

Data collection. Camera-trap data. A total of 73 camera-trap surveys (Supplementary Table 1) were run for $50 \pm 8$ (s.d.) consecutive days on average between 2013 and 2017 at 1,339 locations ( 39 per survey on average) in 32 protected areas (Extended Data Fig. 1) across South Africa. We only used data from protected areas to minimize the effect of humans on temporal activity patterns of herbivores and carnivores ${ }^{37}$. Camera traps were positioned at $\sim 40 \mathrm{~cm}$ above the ground along dirt roads, animal trails and drainage lines commonly used by large mammals ${ }^{38}$. Camera-trap locations were equally distributed across the protected areas to cover different habitats and reduce spatial pseudo-replication (see Extended Data Fig. 7).

These surveys captured 386,815 passages of animals (detections) where species could be identified. From this total dataset, we selected all passages from herbivores $(>2 \mathrm{~kg})$ and larger carnivores $(>20 \mathrm{~kg})$. To reduce the bias obtained by groups or curious individuals triggering the cameras multiple times in a short time period, we defined an independent detection as one detection per species per hour per day at each camera station, yielding 169,771 and 21,259 independent detections for herbivores and carnivores, respectively. We made further selections of these data depending on the analyses as described below.

Survey characteristics. Mean annual rainfall, extracted from climate hazards group infrared precipitation with station data ${ }^{39}$, across the sites was $555 \pm 118 \mathrm{~mm} \mathrm{yr}^{-1}$ and ranged from 287 to $839 \mathrm{~mm} \mathrm{yr}^{-1}$. Mean temperature during the surveys, provided by the South African Weather Service (SAWS, see below), ranged from 11.8 to $27^{\circ} \mathrm{C}$ with a mean of $20.2 \pm 3.5^{\circ} \mathrm{C}$. The following predators were present in the 73 surveys: lion (39), spotted hyena (63), leopard (70), cheetah (49) and wild dog (44) (Supplementary Table 1).

Temperature. Hourly temperature data was provided by the SAWS from 17 permanent weather stations across South Africa that were situated in near the 32 protected areas $\left(\right.$ mean $=50 \mathrm{~km}$, s.d. $=36 \mathrm{~km}$; ArcGIS Desktop 10.5 ${ }^{40}$. To correct for the temperature difference between the protected areas and the SAWS weather stations, we obtained spatial climate data layers from the WorldClim programme (available from http://www.worldclim.org). WorldClim monthly climate data layers are generated through high resolution $(1 \mathrm{~km})$ spatial interpolation of average monthly data on mean temperature between 1960 and $1990^{41}$. We extracted mean monthly temperatures for each protected area and the closest weather station using R v.3.6.0 (ref. ${ }^{42}$ ). We then linearly regressed these monthly temperatures for each protected area and their nearest SAWS weather station, which proved highly accurate $\left(R^{2}>0.99\right)$, and used the estimates from the model (intercept and slope) to correct for temperature differences between each protected area and the nearest SAWS weather station. This yielded approximate hourly temperature data for each survey, from which we calculated the mean temperature per day and per survey. 
We quantified a daily 'timescape of heat' to investigate to what extent herbivores timed activity based on diel changes in temperature. For each survey, we averaged the temperatures across days per hour (Extended Data Fig. 2a,b), and standardized it by rescaling to relative temperatures ranging between 0 and 1 for each survey. This yielded 73 'timescapes of heat' (Extended Data Fig. 2c) with high similarity, with the coolest moments of the day always occurring around 6:00 am and the hottest times during midday (12:00-15:00). The average relative temperature of the 73 timescapes was then used as the final 'timescape of heat' (Fig. 1a). We used this final 'timescape of heat' to quantify the relative temperature of each capture.

Day length. We determined day length for each camera station per day using the maptools package ${ }^{43}$. This package uses algorithms provided by the National Oceanic \& Atmospheric Administration to determine sunrise and sunset given a GPS location and date. We used the time between sunrise and sunset to estimate the proportion of each 24 -h period with daylight for each camera station and date. The day length estimates per camera trap were averaged to get a daily estimate of day length for each survey. We then calculated the mean day length per survey (mean 0.49; range $0.43-0.60$ ) which we used as a covariate in our analyses.

Body mass. Body mass values of the 29 herbivore and five carnivore species were taken as the mean female body mass from Kingdon ${ }^{44}$ (Supplementary Table 2). The preferred body size prey range of lion was based on data by Hayward and Kerley $^{30}$. Note that the authors identified the preferred prey range between 190 and $550 \mathrm{~kg}$. We decreased the lower threshold to $100 \mathrm{~kg}$ on the basis of their data that show that species like common wildebeest $(163 \mathrm{~kg})$ and waterbuck $(186 \mathrm{~kg})$ were preferred. Consistent selection (Jacob's index >0) occurred between 100 and $550 \mathrm{~kg}$ (Extended Data Fig. 5).

Data processing. Activity level. We used the methods developed by Rowcliffe et al. ${ }^{28}$ to estimate activity level for herbivores. This method fits a circular probability density function to the number of observations per hour and estimates the proportion of the day that animals spend active given the assumption that all animals are active at the highest peak in activity. Furthermore, it assumes animals to be active when they trigger the camera. We estimated activity level using the actfit function in the activity package ${ }^{45}$ for each herbivore species in each survey with at least 100 captures per species to increase the robustness of our estimates. This resulted in a subset of 141,883 detections for 29 species that we used for further analyses (Supplementary Table 2). We used a bandwidth adjustment multiplier of 1.5 as suggested by Rowcliffe et al. ${ }^{28}$.

Temporal overlap with carnivores. To estimate the effect of carnivore presence on the activity of herbivores, we calculated a timescape of risk by fitting a circular probability density function to all observations of larger carnivores (lion, spotted hyena, leopard, cheetah and wild dog; $n=21,229$ detections in total). We used the adjusted sun time, calculated using the overlap package ${ }^{46}$, to adjust the time at which animals were recorded by standardizing sunrise at 6:00 and sunset at 18:00. We did this to correct for differences in timing of sunset and sunrise between dates, locations and surveys, enabling comparison among these. We then calculated the overlap between this timescape of risk and activity patterns of herbivores using 1,000 bootstrap iterations and a bandwidth adjustment multiplier of 1.5 in the activity package ${ }^{45}$.

Overlap at sites with and without lions. To further disentangle the effects of predation risk and temperature on herbivore activity patterns, we calculated the overlap between herbivore and lion activity at sites with and without lions. We did not have sufficient data to do this for the other carnivores. They were either present in too many surveys (leopard and spotted hyena), reducing the number of herbivore captures in absence of the carnivore or had low overall number of captures (cheetah and wild dog). We grouped all herbivore observations into daily temperature categories of $2^{\circ} \mathrm{C}$ over the range: $12-14,14-16,16-18,18-20,20-22$, $22-24$ and $24-28^{\circ} \mathrm{C}$ based on the mean temperature for each day. For each of these groups we split the herbivore observations into observations from areas with and without lions. We selected all combinations of species, temperature interval and lion presence/absence with $>100$ observations from the complete dataset, to enable sufficient records for megaherbivores in areas without lions, resulting in 147,027 detections for 24 species. Using these detections, we estimated the activity pattern of each herbivore species per temperature interval for areas with and without lions. Then, we estimated the activity pattern of lions at the different temperature intervals and subsequently calculated the overlap between these and the activity patterns of herbivores as described above using the adjusted sun time and the activity package ${ }^{45}$. We used temperature zone-specific lion activity estimates from areas with lions to estimate overlap with activity patterns of herbivores in the same temperature classes for areas without lions. By comparing overlap in activity patterns within species per temperature category we circumvent potential issues of decreased functioning of the passive infrared sensor of camera traps with increased temperature ${ }^{47}$.

To ensure that potential spatial patterns of lion avoidance would not result in biased sampling of prey activity patterns, we tested for a correlation between the number of lion passages (as a measure of lion activity) and the number of passages of preferred prey (as our sample size for the temporal analyses) per camera station. We used a linear mixed model with a random slope and intercept per survey to account for the nested data of camera stations within surveys. We found no evidence for negative, or positive, spatial associations between visits of lion and their prey (Extended Data Fig. $6 ; F_{1,224}=0.09, P=0.77, n=39$ surveys).

We also tested for possible confounding effects between lion density (predation risk) and climatic factors. There was no significant relationship between lion densities (expressed as the average number of passages per camera per day for each site) and mean annual rainfall (linear regression: $F_{1,15}=2.1, P=0.16$ ) or average temperature (linear regression: $F_{1,15}=0.63, P=0.44$ ). Current lion densities in our South African study sites probably still depend more on management actions, such as the number and year of reintroduction, rather than on environmental conditions.

Statistical analysis. We used GLMM with a beta-regression family to account for data distribution between values of 0 and 1 using the glmmTMB package ${ }^{48}$. For the general trends with body size (Fig. 1 and Supplementary Table 3), we fitted three separate models with relative temperature during activity, overlap with carnivore activity and activity level as response variables and body mass and a second-order term as explanatory variables. Site (protected area) was added as a random factor (intercept) to account for the nested study design (observation within sites). To investigate the effect of lion presence on diel activity patterns of herbivores (Supplementary Table 4), we constructed a GLM with herbivore activity overlap with lion activity as response variable and body mass, including a second-order term, temperature category used to construct the activity overlap (as a continuous variable using the mean for each category) and lion presence as explanatory variables. Daily mean temperature was added as a covariate to account for decreased functioning of the passive infrared sensor of camera traps with increased temperature ${ }^{47}$. We compared models with different combinations of interaction terms and selected the best model based on Akaike Information Criterion (Supplementary Table 6). To further investigate the effect of lion presence on the overlap with lion activity and temperature during activity for individual species (Fig. 2 and Supplementary Table 5), we constructed linear regression models with the difference in overlap and activity temperature between analyses with and without lion presence as response variables and body size and its second-order term as explanatory variables. All statistics were performed in R v.3.6.0 (ref. ${ }^{42}$ ).

Reporting Summary. Further information on research design is available in the Nature Research Reporting Summary linked to this article.

\section{Data availability}

The data are located on the Dryad Digital Repository: https://doi.org/10.5061/ dryad.6m905qfvx.

\section{Code availability}

$\mathrm{R}$ code of all analyses is available via the Dryad Digital Repository: https://doi. org/10.5061/dryad.6m905qfvx.

Received: 11 October 2019; Accepted: 4 May 2020;

Published online: 1 June 2020

\section{References}

1. Ripple, W. J. et al. Saving the world's terrestrial megafauna. Bioscience 66 , 807-812 (2016).

2. Daskin, J. H. \& Pringle, R. M. Warfare and wildlife declines in Africa's protected areas. Nature 553, 328-332 (2018).

3. Craigie, I. D. et al. Large mammal population declines in Africa's protected areas. Biol. Conserv. 143, 2221-2228 (2010).

4. Pacifici, M., Visconti, P. \& Rondinini, C. A framework for the identification of hotspots of climate change risk for mammals. Glob. Change Biol. 24, 1626-1636 (2018)

5. Thuiller, W. et al. Vulnerability of African mammals to anthropogenic climate change under conservative land transformation assumptions. Glob. Change Biol. 12, 424-440 (2006).

6. Moritz, C. \& Agudo, R. The future of species under climate change: resilience or decline? Science 341, 504-508 (2013).

7. Harris, G., Thirgood, S. J., Hopcraft, J. G. C., Cromsigt, J. P. G. M. \& Berger, J. Global decline in aggregated migrations of large terrestrial mammals. Endang. Species Res. 7, 55-76 (2009).

8. Pekor, A. et al. Fencing Africa's protected areas: costs, benefits, and management issues. Biol. Conserv. 229, 67-75 (2019).

9. Crooks, K. R. et al. Quantification of habitat fragmentation reveals extinction risk in terrestrial mammals. Proc. Natl Acad. Sci. USA 114, 7635-7640 (2017)

10. Engelbrecht, F. et al. Projections of rapidly rising surface temperatures over Africa under low mitigation. Environ. Res. Lett. 10, 085004 (2015).

11. Hetem, R. S., Fuller, A., Maloney, S. K. \& Mitchell, D. Responses of large mammals to climate change. Temperature 1, 115-127 (2014). 
12. Fuller, A., Mitchell, D., Maloney, S. K. \& Hetem, R. S. Towards a mechanistic understanding of the responses of large terrestrial mammals to heat and aridity associated with climate change. Clim. Change Responses $\mathbf{3}$, 10 (2016).

13. Pinsky, M. L., Eikeset, A. M., McCauley, D. J., Payne, J. L. \& Sunday, J. M. Greater vulnerability to warming of marine versus terrestrial ectotherms. Nature 569, 108-111 (2019).

14. Hetem, R. S. et al. Activity re-assignment and microclimate selection of free-living Arabian oryx: responses that could minimise the effects of climate change on homeostasis? Zoology 115, 411-416 (2012).

15. Huey, R. B. \& Tewksbury, J. J. Can behavior douse the fire of climate warming? Proc. Natl Acad. Sci. USA 106, 3647-3648 (2009).

16. McCain, C. M. \& King, S. R. B. Body size and activity times mediate mammalian responses to climate change. Glob. Change Biol. 20, 1760-1769 (2014).

17. Kohl, M. T. et al. Diel predator activity drives a dynamic landscape of fear. Ecol. Monogr. 88, 638-652 (2018).

18. Levy, O., Dayan, T., Porter, W. P. \& Kronfeld-Schor, N. Time and ecologica resilience: can diurnal animals compensate for climate change by shifting to nocturnal activity? Ecol. Monogr. 89, e01334 (2019).

19. Milling, C. R., Rachlow, J. L., Johnson, T. R., Forbey, J. S. \& Shipley, L. A. Seasonal variation in behavioral thermoregulation and predator avoidance in a small mammal. Behav. Ecol. 28, 1236-1247 (2017).

20. Tambling, C. J. et al. Temporal shifts in activity of prey following large predator reintroductions. Behav. Ecol. Sociobiol. 69, 1153-1161 (2015).

21. Veldhuis, M. P. et al. Large herbivore assemblages in a changing climate: incorporating water dependence and thermoregulation. Ecol. Lett. 22 1536-1546 (2019).

22. Terrien, J., Perret, M. \& Aujard, F. Behavioral thermoregulation in mammals: a review. Front. Biosci. 16, 1428-1444 (2011).

23. Sinclair, A. R. E., Mduma, S. \& Brashares, J. S. Patterns of predation in a diverse predator-prey system. Nature 425, 288-290 (2003).

24. Owen-Smith, N. Megaherbivores: The Influence of Very Large Body Size on Ecology (Cambridge Univ. Press, 1988).

25. McCafferty, D. J. et al. Estimating metabolic heat loss in birds and mammals by combining infrared thermography with biophysical modelling. Comp. Biochem. Physiol. A 158, 337-345 (2011).

26. Clauss, M., Steuer, P., Müller, D. W. H., Codron, D. \& Hummel, J. Herbivory and body size: allometries of diet quality and gastrointestinal physiology, and implications for herbivore ecology and dinosaur gigantism. PLOS ONE 8, $1-16$ (2013).

27. Kronfeld-Schor, N., Visser, M. E., Salis, L. \& van Gils, J. A. Chronobiology of interspecific interactions in a changing world. Phil. Trans. R. Soc. B 372, 20160248 (2017)

28. Rowcliffe, J. M., Kays, R., Kranstauber, B., Carbone, C. \& Jansen, P. A. Quantifying levels of animal activity using camera trap data. Methods Ecol. Evol. 5, 1170-1179 (2014).

29. Porter, W. P. \& Kearney, M. Size, shape, and the thermal niche of endotherms. Proc. Natl Acad. Sci. USA 106, 19666-19672 (2009).

30. Hayward, M. W. \& Kerley, G. I. H. Prey preferences of the lion (Panthera leo). J. Zool. 267, 309-322 (2005).

31. Tambling, C. J. et al. Spatial and temporal changes in group dynamics and range use enable anti-predator responses in African buffalo. Ecology 93, 1297-1304 (2012).

32. Hayward, M. W. \& Kerley, G. I. H. Prey preferences and dietary overlap amongst Africa's large predators. S. Afr. J. Wildl. Res. 38, 93-108 (2008).

33. Kinahan, A. A., Pimm, S. L. \& van Aarde, R. J. Ambient temperature as a determinant of landscape use in the savanna elephant, Loxodonta africana. J. Therm. Biol. 32, 47-58 (2007).

34. le Roux, E., Kerley, G. I. H. \& Cromsigt, J. P. G. M. Megaherbivores modify trophic cascades triggered by fear of predation in an African savanna ecosystem. Curr. Biol. 28, 2493-2499 (2018).
35. Atkins, J. L. et al. Cascading impacts of large-carnivore extirpation in an African ecosystem. Science 364, 173-177 (2019).

36. Bonnot, N. C. et al. Sitka black-tailed deer (Odocoileus hemionus sitkensis) adjust habitat selection and activity rhythm to the absence of predators. Can. J. Zool. 94, 385-394 (2016).

37. Gaynor, K. M., Hojnowski, C. E., Carter, N. H. \& Brashares, J. S. The influence of human disturbance on wildlife nocturnality. Science (80-) 360, 1232 LP-1235 (2018).

38. Miller, J. R. B., Pitman, R. T., Mann, G. K. H., Fuller, A. K. \& Balme, G. A Lions and leopards coexist without spatial, temporal or demographic effects of interspecific competition. J. Anim. Ecol. 87, 1709-1726 (2018).

39. Funk, C. et al. The climate hazards infrared precipitation with stations-a new environmental record for monitoring extremes. Sci. Data 2, 150066 (2015)

40. ArcGIS Desktop: Release 10.5 (ESRI, 2015).

41. Hijmans, R. J., Cameron, S. E., Parra, J. L., Jones, P. G. \& Jarvis, A. Very high resolution interpolated climate surfaces for global land areas. Int. J. Climatol. 25, 1965-1978 (2005)

42. R Core Team R: A Language and Environment for Statistical Computing (R Foundation for Statistical Computing, 2018).

43. Bivand, R. \& Lewin-Koh, N. maptools: Tools for handling spatial objects. R package version 0.9-5 (2019).

44. Kingdon, J. et al. Mammals of Africa (Bloomsbury, 2013).

45. Rowcliffe, M. activity: Animal activity statistics. R package version 1.2 (2019)

46. Ridout, M. S. \& Linkie, M. Estimating overlap of daily activity patterns from camera trap data. J. Agric. Biol. Environ. Stat. 14, 322-337 (2009).

47. Hofmeester, T. R. et al. Framing pictures: a conceptual framework to identify and correct for biases in detection probability of camera traps enabling multi-species comparison. Ecol. Evol. 9, 2320-2336 (2019).

48. Brooks, M. E. et al. glmmTMB balances speed and flexibility among packages for zero-inflated generalized linear mixed modeling. $R$ J. 9, 378-400 (2017).

\section{Acknowledgements}

We thank the Ezemvelo KZN Wildlife management and research staff for their help and logistical support while undertaking this study. The camera-trap surveys were funded by Panthera (with support from Peace Parks Foundation and Cartier) and run with the help of staff and volunteers from Wildlife ACT and Siyafunda Conservation. Furthermore, M.P.V. has been financially supported by the AfricanBioServices project which received funding from the European Union's Horizon 2020 research and innovation programme under grant no. 641918. We thank J. L. Atkins, R. S. Hetem, H. Olff, N. Owen-Smith and R. M. Pringle for their comments on earlier versions of the manuscript.

\section{Author contributions}

M.P.V. and J.P.M.G.C. conceived the study and developed the concept. G.B., R.T.P. and D.J.D. contributed data. T.R.H. and M.P.V. analysed the data. M.P.V. and J.P.M.G.C. wrote the first draft of the manuscript and all authors contributed revisions.

\section{Competing interests}

The authors declare no competing interests.

\section{Additional information}

Extended data is available for this paper at https://doi.org/10.1038/s41559-020-1218-2. Supplementary information is available for this paper at https://doi.org/10.1038/ s41559-020-1218-2.

Correspondence and requests for materials should be addressed to M.P.V

Reprints and permissions information is available at www.nature.com/reprints. Publisher's note Springer Nature remains neutral with regard to jurisdictional claims in published maps and institutional affiliations.

(c) The Author(s), under exclusive licence to Springer Nature Limited 2020 


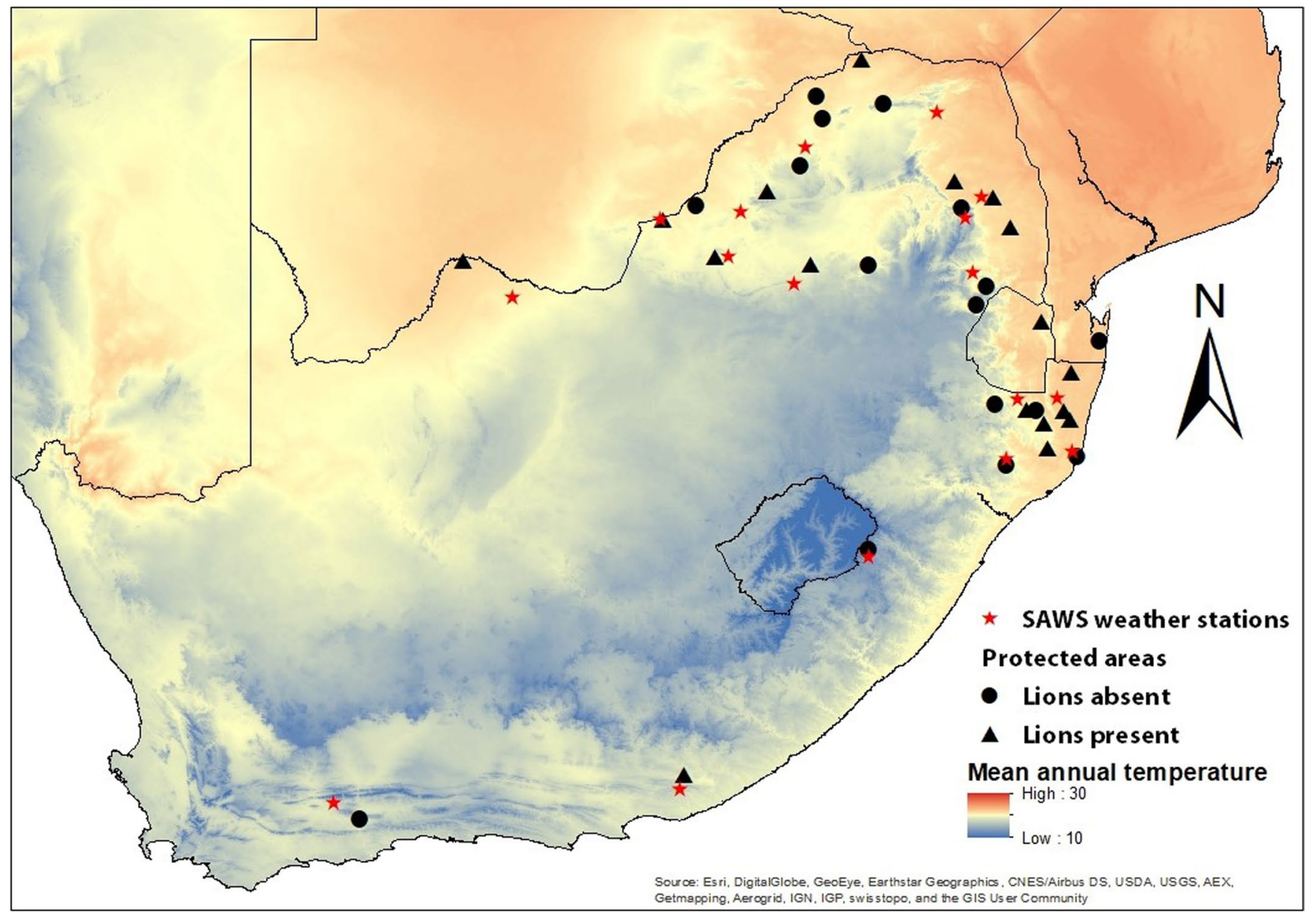

Extended Data Fig. 1 | Overview of 32 protected areas in South Africa with camera-trap surveys and 17 South African Weather Service stations. Background colour represent mean annual temperature (WorldClim.org). Lions were either present (circles) or absent (triangle). 

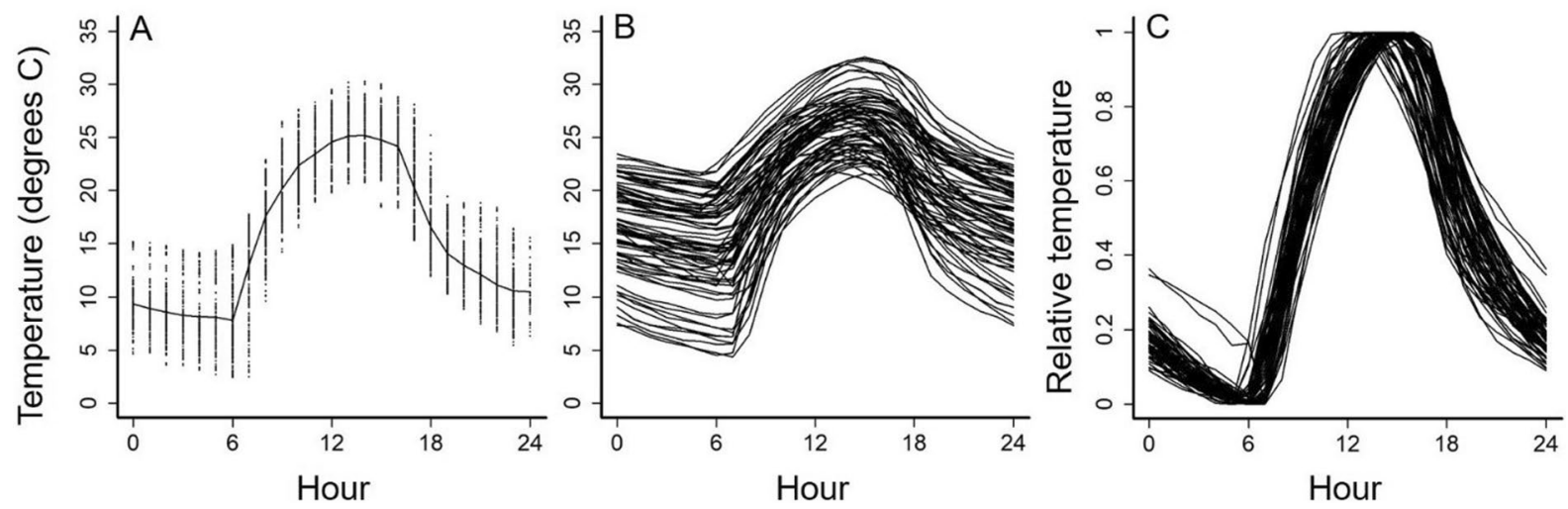

Extended Data Fig. 2 | Daily temperature distributions during the 73 camera-trap surveys in 32 protected areas in South Africa. A, hourly temperature records for an example survey (Zingela 2017). Line represents the hourly mean, dots are individual observations. B, temperature averaged by hour for the 73 surveys and $\mathbf{C}$, relative temperature for each survey (standardized between 0 and 1 ). 


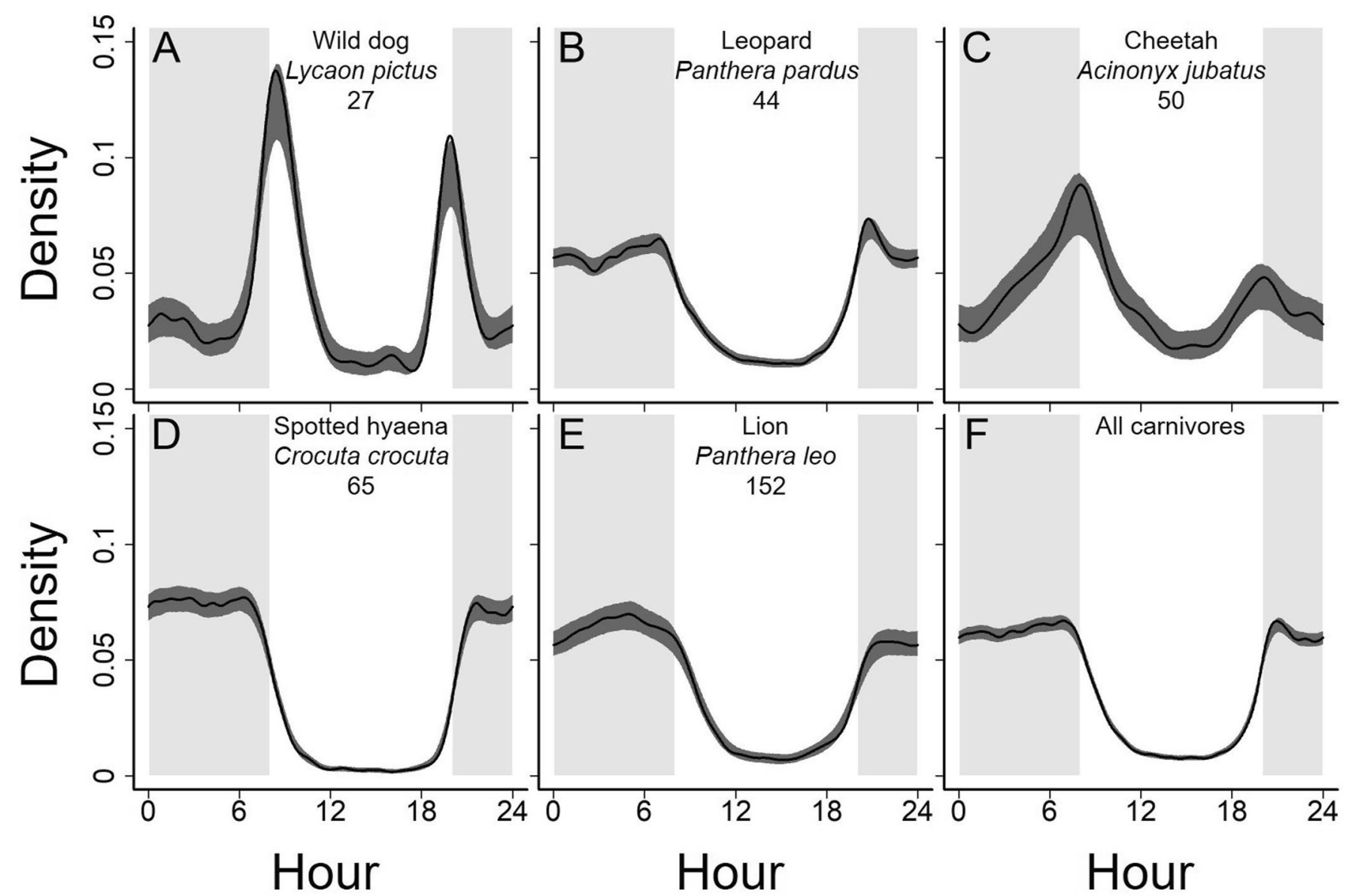

Extended Data Fig. 3 | Diel activity patterns of larger carnivores. Wild dog ( $\mathbf{A} ; n=751$ detections), leopard $(n=6,833$ detections), cheetah ( $n=487$ detections), spotted hyena ( $n=5,331$ detections), lion ( $n=2,657$ detections) and all carnivores combined $(\mathbf{F} ; n=16,059$ detections). Species are ordered by increasing body mass, which is presented in kilograms below the scientific name. Dark grey represents $95 \%$ confidence interval around the estimated activity pattern, grey background represents period of high carnivore activity. 


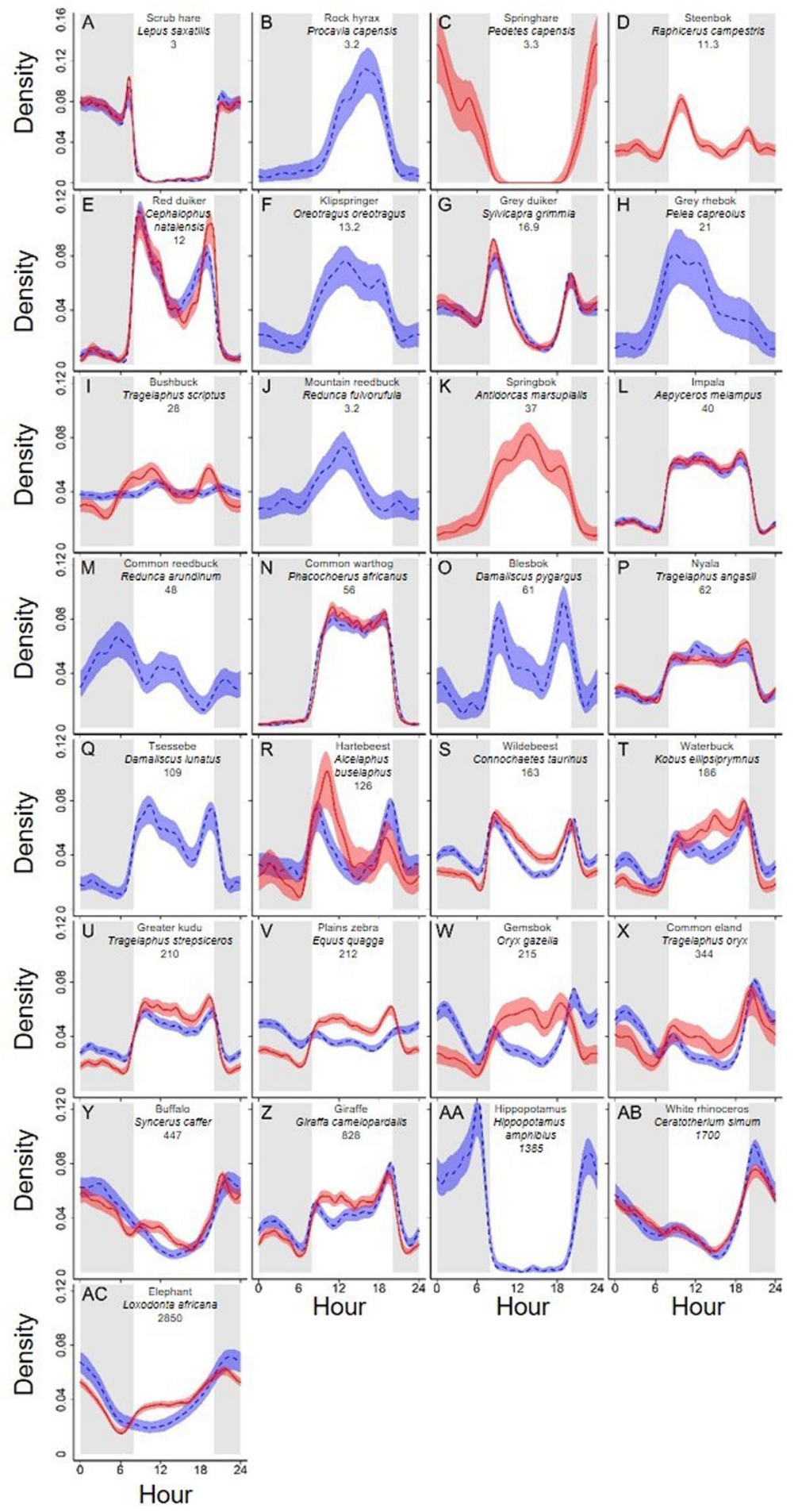

Extended Data Fig. 4 | Diel activity patterns of the $\mathbf{2 9}$ herbivores in protected areas with lions (red) or without lions (blue). Species are ordered by increasing body mass, which is presented in kilograms below the scientific name. Grey background represents period of high carnivore activity (Extended Data Fig. 3). Coloured area around the estimates represents the $95 \%$ confidence interval. 


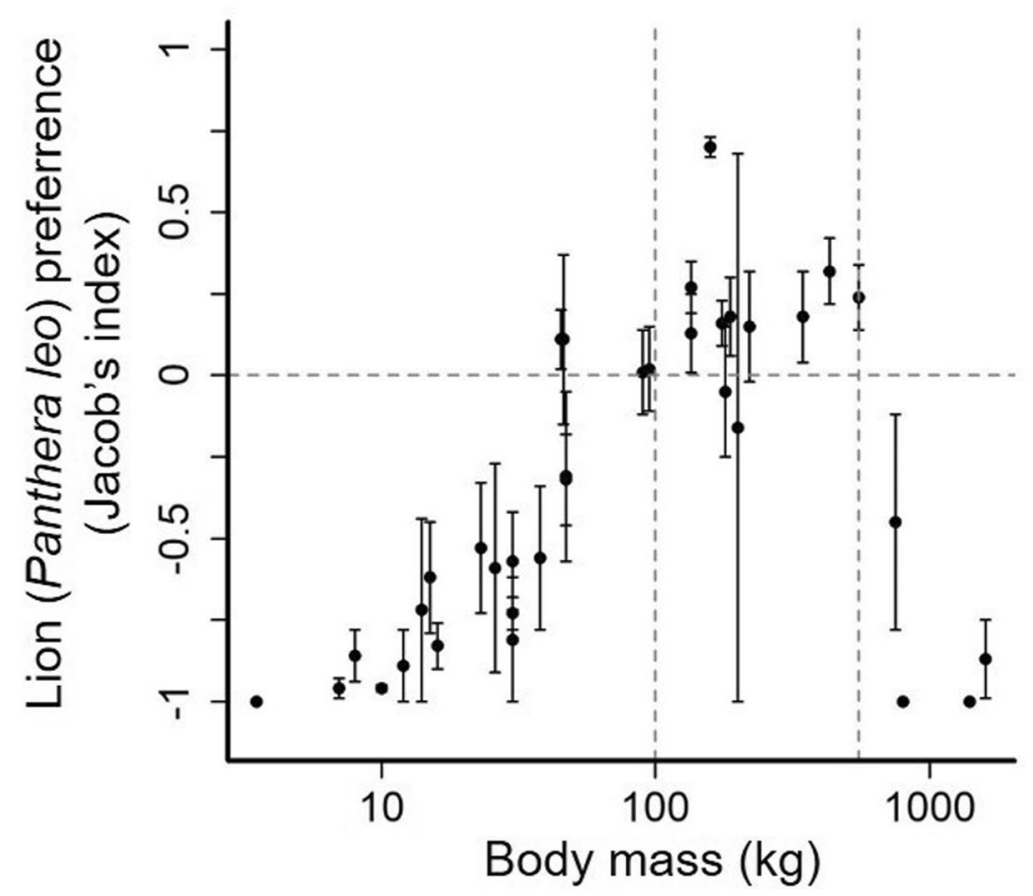

Extended Data Fig. 5 | Lion (Panthera leo) dietary preferences based on Jacobs' index (mean \pm SE) of 48 lion populations across Africa at differing prey densities. Data from ref. ${ }^{30}$. Only species recorded in lion diet more than once were included in the dataset. Vertical lines represent the preferred prey range used in this study $(100-550 \mathrm{~kg})$. 


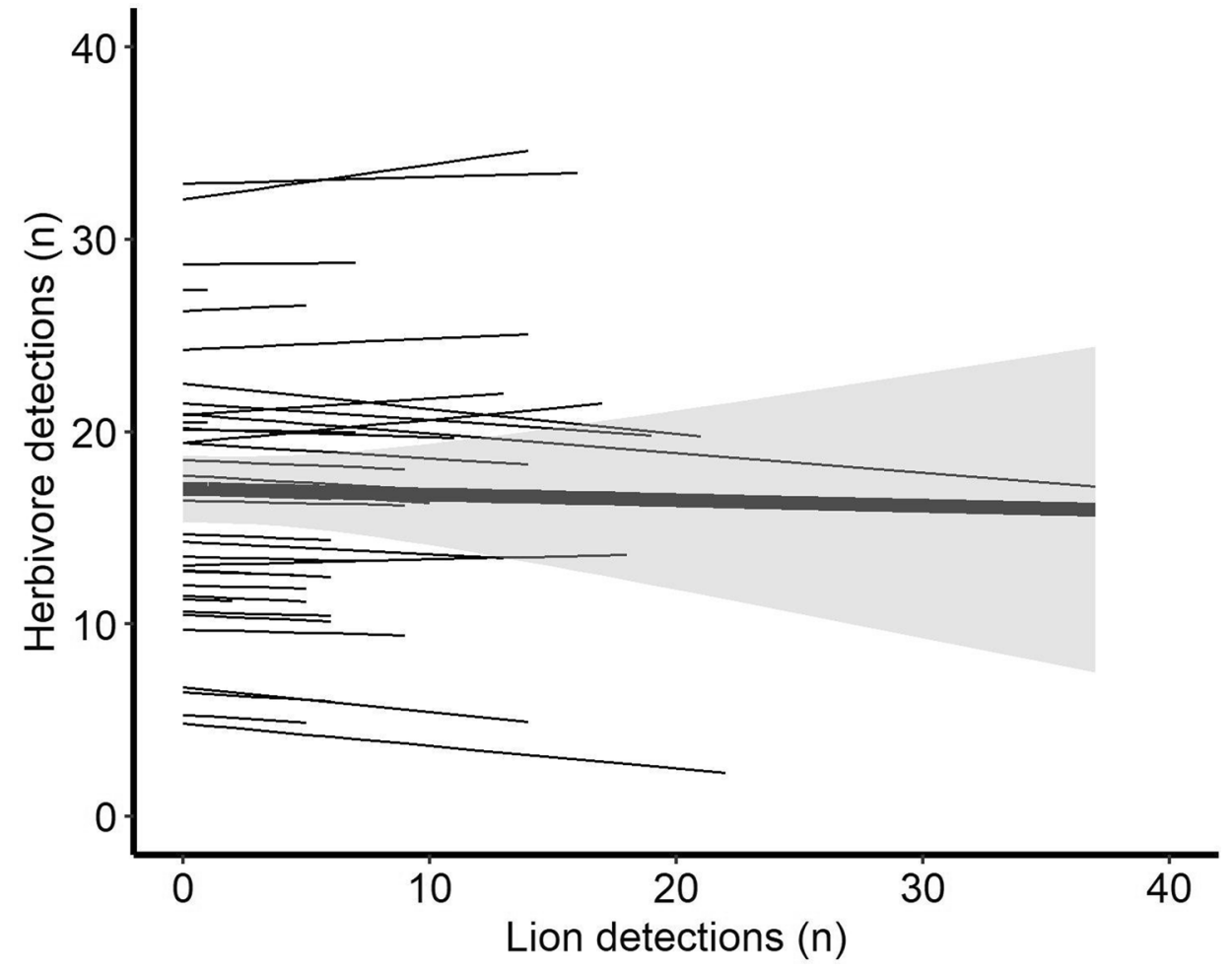

Extended Data Fig. 6 | Herbivore detections at each camera-trap location are not related to the number of lion detections. (Linear Mixed Model: $F_{1,22.4}=0.09, P=0.77, n=39$ surveys). Only herbivore species in the preferred prey range of lion (100-550kg) were included in this analysis. 


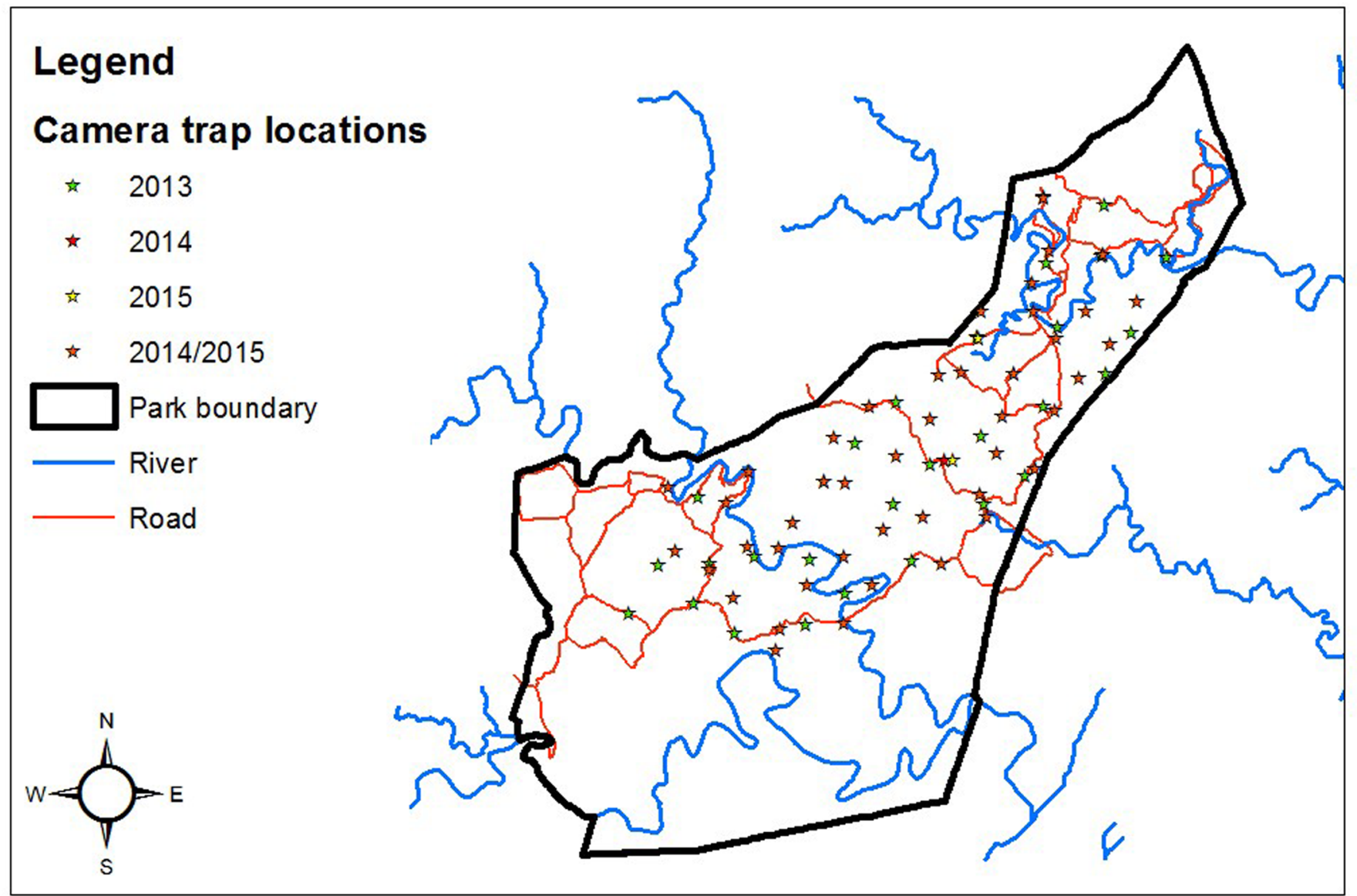

Extended Data Fig. 7 | Camera-trap locations for Hluhluwe-iMfolozi Park. An example of how cameras were distributed across protected areas. The most Southern part of the area is managed as a wilderness area where tourist and research access is limited and was thus excluded in this study. 


\section{Reporting Summary}

Nature Research wishes to improve the reproducibility of the work that we publish. This form provides structure for consistency and transparency in reporting. For further information on Nature Research policies, see Authors \& Referees and the Editorial Policy Checklist.

\section{Statistics}

For all statistical analyses, confirm that the following items are present in the figure legend, table legend, main text, or Methods section.

$\mathrm{n} / \mathrm{a}$ | Confirmed

$\square$ \.he exact sample size $(n)$ for each experimental group/condition, given as a discrete number and unit of measurement

$\square$ \ A statement on whether measurements were taken from distinct samples or whether the same sample was measured repeatedly

The statistical test(s) used AND whether they are one- or two-sided

$\triangle$ Only common tests should be described solely by name; describe more complex techniques in the Methods section.

$\square$ \ A description of all covariates tested

$\square$ \ A description of any assumptions or corrections, such as tests of normality and adjustment for multiple comparisons

$\square$ A full description of the statistical parameters including central tendency (e.g. means) or other basic estimates (e.g. regression coefficient) AND variation (e.g. standard deviation) or associated estimates of uncertainty (e.g. confidence intervals)

$\square$ For null hypothesis testing, the test statistic (e.g. $F, t, r$ ) with confidence intervals, effect sizes, degrees of freedom and $P$ value noted

$\square$ Give P values as exact values whenever suitable.

Х $\square$ For Bayesian analysis, information on the choice of priors and Markov chain Monte Carlo settings

$\square$ \ For hierarchical and complex designs, identification of the appropriate level for tests and full reporting of outcomes

$\bigotimes \square$ Estimates of effect sizes (e.g. Cohen's $d$, Pearson's $r$ ), indicating how they were calculated

Our web collection on statistics for biologists contains articles on many of the points above.

\section{Software and code}

Policy information about availability of computer code

Data collection No software used to collect data

Data analysis All data were processed and analyzed using R3.6.0

For manuscripts utilizing custom algorithms or software that are central to the research but not yet described in published literature, software must be made available to editors/reviewers. We strongly encourage code deposition in a community repository (e.g. GitHub). See the Nature Research guidelines for submitting code \& software for further information.

\section{Data}

Policy information about availability of data

All manuscripts must include a data availability statement. This statement should provide the following information, where applicable:

- Accession codes, unique identifiers, or web links for publicly available datasets

- A list of figures that have associated raw data

- A description of any restrictions on data availability

The data are located on the Dryad Digital Repository (doi.org/10.5061/dryad.6m905afvx)

\section{Field-specific reporting}

Please select the one below that is the best fit for your research. If you are not sure, read the appropriate sections before making your selection. $\bigotimes$ Life sciences $\quad \square$ Behavioural \& social sciences $\square$ Ecological, evolutionary \& environmental sciences 


\section{Life sciences study design}

All studies must disclose on these points even when the disclosure is negative.

Sample size No sample-size calculation was performed. We used camera trap data from 32 different protected areas in which about half lions were present. This is far beyond the sample sizes used in any study so far on herbivore behavior in response to predation risk. To calculate activitity patterns for each herbivore species, we set the sample size threshold at 100 captures per species per survey to increase the robustness of our estimates. Decreasing this threshold did not qualitatively change our results.

Data exclusions We set the sample size threshold at 100 captures per species per survey to increase the robustness of our estimates. Decreasing this threshold did not qualitatively change our results. Thus, species with less than 100 captures were excluded.

Replication The design included replication of predator presence and absence, with 16 areas (replications) of lion presence and 16 areas (replications) of lion absence.

Randomization Allocation of predator or herbivore presence was not done randomly, but based on natural occurence of herbivores and reintroduction of lions in protected area across South Africa. Nevertheless, this comes very close to a "natural experiment" as lions used to be present in all 32 protected areas and there is no bias in environmental characteristics (temperature, rainfall) between areas with and without lions present.

Blinding

Data collection was done via camera traps so this could be seen as "blind" data collection for the purpose of this study.

\section{Reporting for specific materials, systems and methods}

We require information from authors about some types of materials, experimental systems and methods used in many studies. Here, indicate whether each material, system or method listed is relevant to your study. If you are not sure if a list item applies to your research, read the appropriate section before selecting a response.

\begin{tabular}{|c|c|}
\hline $\mathrm{n} / \mathrm{a}$ & Involved in the study \\
\hline Х & $\square$ Antibodies \\
\hline$凶$ & $\square$ Eukaryotic cell lines \\
\hline 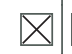 & $\square$ Palaeontology \\
\hline 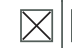 & $\square$ Animals and other organisms \\
\hline$\bigotimes$ & $\square$ Human research participants \\
\hline Х & $\square$ Clinical data \\
\hline
\end{tabular}

\begin{tabular}{l|l} 
Methods \\
\hline n/a & Involved in the study \\
$\square$ & $\square$ ChIP-seq \\
$\square$ & $\square$ Flow cytometry \\
$\square$ & $\square$ MRI-based neuroimaging
\end{tabular}

